\title{
Relationship between Unusual Hepatic Acyl Coenzyme A Profiles and the Pathogenesis of Reye Syndrome
}

\author{
Barbara E. Corkey, ${ }^{\star}$ Daniel E. Hale, ${ }^{*}$ M. Clay Glennon, ${ }^{3}$ Richard I. Kelley, ${ }^{\ddagger}$ Paul M. Coates, ${ }^{\ddagger}$ Laurie Kilpatrick, ${ }^{\ddagger}$ \\ and Charles A. Stanley" \\ ${ }^{*}$ Division of Diabetes and Metabolism, Boston University School of Medicine, Boston, Massachusetts 02118; and ${ }^{\ddagger}$ Departments of \\ Pediatrics and ${ }^{\S}$ Biochemistry and Biophysics, University of Pennsylvania School of Medicine, Philadelphia, Pennsylvania 19104
}

\begin{abstract}
This study examines the relationship between impaired fatty acid oxidation and the pathogenesis of Reye syndrome. We present a hypothesis proposing that many clinical signs of this childhood disease are caused by accumulation of unusual acyl CoA esters, precursors to deacylated metabolites found in the patients' blood and urine. A new method was developed to measure acyl $\mathrm{CoA}$ compounds in small human liver biopsy samples, offering several advantages over previous techniques. A major finding was an accumulation in Reye syndrome patients of short- and medium-chain acyl $\mathrm{CoA}$ intermediates of fatty acid and branched-chain amino acid oxidation. These metabolites included octanoyl, isovaleryl, butyryl, isobutyryl, propionyl, and methylmalonyl $\mathrm{CoA}$ esters. The findings were explained in a model of hepatic fatty acid oxidation involving three interrelated pathways: mitochondrial $\beta$-oxidation, peroxisomal $\beta$-oxidation, and $\omega$-oxidation in the endoplasmic reticulum. The results suggest that pathogenesis in Reye syndrome stems from generalized mitochondrial damage resulting in accumulation of acyl CoA esters. High levels of these compounds lead to inhibition of mitochondrial pathways for ureogenesis, gluconeogenesis, and fatty acid oxidation. The inhibited pathways, in turn, could cause the hyperammonemia, hypoglycemia, and hypoketonemia observed in patients. The model also explains underlying biochemical differences between patients with Reye syndrome and medium-chain acyl CoA dehydrogenase deficiency, another disorder of fatty acid metabolism. Acetyl CoA levels, in the latter disease, were dramatically decreased, compared with both human controls and Reye syndrome patients.
\end{abstract}

\section{Introduction}

For nearly 25 years, researchers have sought the cause of Reye syndrome, an often fatal disease that strikes children after a viral illness (1-5). In recent years, many investigators have focused their search on the liver, in which disturbances in lipid

A preliminary report of this work was presented at the Annual Meeting of the Society for Inherited Metabolic Disorders, Seabrook Island, SC, 9-12 March 1986.

Address reprint requests to Dr. Barbara E. Corkey, E-205, Division of Diabetes and Metabolism, Evans Department of Medicine, Boston University School of Medicine, Boston, MA 02118.

Received for publication 28 March 1987 and in revised form 29 March 1988.

J. Clin. Invest.

(C) The American Society for Clinical Investigation, Inc.

0021-9738/88/09/0782/07 \$2.00

Volume 82, September 1988, 782-788 metabolism may play a role in either the cause or the pathology of the disease.

One of the primary characteristics of Reye syndrome is an abnormally high level of circulating FFA (1-5). Other disturbances point directly to impaired fatty acid oxidation in the liver. For example, blood levels of $\beta$-hydroxybutyrate and acetoacetate, end products of $\beta$-oxidation by liver cells, are depressed in these patients (2). The patients' urine also contains unusual short- and medium-chain ${ }^{1}$ fatty acids and their metabolites $(2,6,7)$; the former compounds normally are oxidized in the liver. Finally, hepatocytes show an increase in both lipid droplets and peroxisomes (1-5). Peroxisomes are secondary sites of fatty acid oxidation $(8,9)$.

How might these perturbations in fatty acid oxidation be involved in the pathology of Reye syndrome? A clear causal relationship has not been established, but at least one intriguing hypothesis exists. The unusual fatty acids excreted by Reye patients, such as propionate, butyrate, and octanoate, are derived from acyl CoA precursors. As these precursors accumulate, they could compete for enzyme-binding sites with acetyl CoA or other acyl CoA substrates, profoundly disrupting normal biochemical pathways for energy metabolism and ureogenesis (10-12).

To explore this hypothesis, we developed a sensitive method to measure the short- and medium-chain acyl CoA esters in liver biopsy specimens from patients with Reye syndrome. The method, never before applied to human tissue, also was used on control subjects undergoing liver biopsy for other reasons. A third group of patients with medium-chain acyl CoA dehydrogenase (MCAD) ${ }^{2}$ deficiency was evaluated. In this congenital disease, one of the essential enzymes of mitochondrial $\beta$-oxidation is missing, causing many of the same signs as Reye syndrome $(13,14)$.

The goal of this research was to determine whether or not acyl CoA esters, possible inhibitors of several metabolic pathways, accumulate in livers of patients with Reye syndrome. The measurements may be correlated with the deacylated metabolites previously found in the blood and urine of these patients and with clinical manifestations of the disease.

Another objective was to pinpoint the biochemical defects responsible for altered fatty acid metabolism in Reye syndrome. Finally, the measurements may illuminate, on a biochemical level, further differences between Reye syndrome and MCAD deficiency, diseases that can be hard to differentiate clinically (15).

1. Fatty acids and acyl CoA esters in this text are defined by their carbon chain length as short, $2-6$; medium; $6-14$; or long, 14 or more carbons.

2. Abbreviations used in this paper: MCAD, medium-chain acyl CoA dehydrogenase; TNF, tumor necrosis factor. 
This report demonstrates abnormal acyl CoA profiles in patients with Reye syndrome. It also presents a hypothesis to explain how these unusual metabolites may be involved in the pathogenesis of Reye syndrome. A general model also was developed to show the cooperative interactions of the three pathways of hepatic fatty acid oxidation: mitochondrial $\beta$-oxidation, peroxisomal $\beta$-oxidation, and microsomal $\omega$-oxidation.

\section{Methods}

Patients. Patients with Reye syndrome were identified by criteria set forth by the Communicable Disease Center (2). Informed consent for participation in this study was obtained from the parents or guardians of patients undergoing diagnostic liver biopsy. The additional portion of liver used for this study entailed no additional risk for the patient. The clinical characteristics of these patients are shown in Table I. The two patients with MCAD deficiency and one control subject (C-1) have been described previously $(13,14)$. Four additional subjects (Table II) were subjected to liver biopsy in the course of establishing diagnoses of chronic hepatitis (C-2), hypopituitarism (C-3), Hodgkin's lymphoma (C-4), and muscular dystrophy (C-5). The control, C-5, had been fasted overnight before biopsy and was previously evaluated for acyl CoA dehydrogenase deficiency and found to have normal enzyme levels.

Sample preparation. Because of the small samples available from human subjects, a decision was made to measure short- and mediumchain fatty acyl CoA esters, rather than long-chain compounds. This choice was based on two main considerations. First, a previous study by Kang et al. (16) showed no differences in levels of long-chain acyl CoA compounds in necropsy liver samples, between control subjects and Reye syndrome patients. Second, abnormal urinary metabolites in these patients are derived from short- and medium-chain compounds, not long-chain esters.

Samples were obtained by needle biopsy, frozen in liquid nitrogen within 2 min after removal from the patient, and stored at $-70^{\circ} \mathrm{C}$. Samples were prepared for analysis as follows: $10-20 \mathrm{mg}$ frozen tissue was rapidly weighed and then extracted with $0.4 \mathrm{ml}$ of ice-cold $0.6 \mathrm{~N}$ TCA. Each sample was briefly sonicated in an ice bath until a homogeneous mixture was obtained, using $\sim 10$ bursts of $1-3 \mathrm{~s}$ with a microtip at a setting of 4 (model W185 F sonicator cell disrupter; Heat Systems-Ultrasonics, Inc., Plainview, NY). The acid-insoluble residue was removed by centrifugation at $12,000 \mathrm{~g}$ for $30 \mathrm{~s}$. These pellets were washed several times with ether, dried overnight, and weighed to determine fat-free dry weight. Dry weight averaged $17.8 \pm 2.2 \%$ (SD) of wet weight and was similar in all samples. The supernatant was neutralized and the TCA was removed by vigorous vortexing with $0.6 \mathrm{ml}$

Table I. Clinical Description of Patients with Reye Syndrome

\begin{tabular}{lccc}
\hline \multicolumn{1}{c}{ Patient } & 1 & 2 & 3 \\
\hline Age $(y r / m o)$ & $10 \mathrm{yr} / 3 \mathrm{mo}$ & $11 \mathrm{yr} / 9 \mathrm{mo}$ & $7 \mathrm{yr} / 10 \mathrm{mo}$ \\
Sex & F & F & M \\
Prodromal illness & URI & URI & Varicella \\
Aspirin used & Yes & Yes & Yes \\
Ammonia $(\mu g / d l)$ & 262 & 294 & 243 \\
SGOT $(I U)$ & 369 & 53 & 550 \\
SGPT $(I U)$ & 783 & 110 & 695 \\
Coma stage & III & II & IV \\
Time of biopsy (hours & & & \\
after admission) & 7.5 & 7 & 4 \\
Outcome & Survived & Died & Survived \\
\hline
\end{tabular}

Abbreviations: SGOT, serum glutamic oxaloacetic transaminase; SGPT, serum glutamic pyruvic transaminase.
Table II. Clinical Description of Control Subjects

\begin{tabular}{cccrl}
\hline Patient & Age & Sex & Fast & \multicolumn{1}{c}{ Diagnosis } \\
\hline & $y r / m o$ & & $h$ & \\
1 & $11 / 3$ & M & 0 & Heterozygote for MCAD \\
2 & $12 / 1$ & F & 0 & Chronic hepatitis \\
3 & $18 / 7$ & M & 0 & Hypopituitarism \\
4 & $16 / 9$ & F & 0 & Hodgkin's lymphoma (normal liver) \\
5 & $6 / 6$ & M & 15 & Muscular dystrophy \\
\hline
\end{tabular}

of diethyl ether. After centrifugation at $12,000 \mathrm{~g}$ for $30 \mathrm{~s}$, the upper phase containing the ether and TCA was totally removed. This procedure was repeated about five times until the $\mathrm{pH}$ of the extract reached 6.0. The sample was then evaporated to dryness and stored at $-70^{\circ} \mathrm{C}$ until assayed.

Acyl CoA analysis. Profiles of acid-soluble acyl CoA esters were obtained using a modification of the HPLC method of Corkey et al. (17) with a dual pump system (model 324; Beckman Instruments, Fullerton, CA or Waters Associates, Milford, MA). Absorbance measurements were made at $254 \mathrm{~nm}$ at a sensitivity of 0.005 AUFS using a $5-\mu \mathrm{m} \mathrm{C}_{18}$ reverse-phase column (Novapak; Waters Associates). The published method, using three separate isocratic separations and methanol as organic solvent (17), was used to evaluate acyl CoA esters in patients $C-1, M-1$, and $M-2$, and was found to be inadequate for measuring small samples. The modified method permitted analysis of all acid-soluble esters in as little as $10 \mathrm{mg}$ (wet weight) of tissue. Comparable values were obtained from rat liver samples measured by both techniques (data not shown). The mobile phases used in the modified procedure were $0.1 \mathrm{M} \mathrm{KH}_{2} \mathrm{PO}_{4}, \mathrm{pH} 5.0$ (buffer A) and $0.1 \mathrm{M} \mathrm{KH}_{2} \mathrm{PO}_{4}$ containing $40 \%$ acetonitrile, pH 5.0 (buffer $\mathrm{B}$ ). Elution of acid-soluble CoA esters from malonyl CoA to octanoyl CoA was achieved by varying both the gradient and the rate of flow. The initial composition of the mobile phase was $7.5 \%$ buffer B. This was increased to $10 \%$ at 22 $\mathrm{min}$ (over $1 \mathrm{~min}$ ) and to $80 \%$ at $50 \mathrm{~min}$ (over $40 \mathrm{~min}$ ). The initial flow rate was $0.4 \mathrm{ml} / \mathrm{min}$. This was increased to $0.8 \mathrm{ml} / \mathrm{min}$ at $20 \mathrm{~min}$ (over $1 \mathrm{~min}$ ) and to $1.2 \mathrm{ml} / \mathrm{min}$ at $55 \mathrm{~min}$ (over $5 \mathrm{~min}$ ).

Identification of peaks was based on three criteria: $(a)$ retention times of standards injected separately; $(b)$ demonstration that acyl CoA compounds, in samples, increased by a predictable amount when the sample was rechromatographed with added standard; and (c) demonstration that identified peaks disappeared after alkaline hydrolysis with a compensatory increase in free CoASH plus glutathione CoA. The second or third identification procedure was performed on 4 of the 10 human samples. The second procedure also verified that sample extracts did not alter elution times or peak areas. Quantitation of acyl CoA esters was based on comparison of sample peak areas with those obtained from standards (see reference 17 for detailed discussion of peak identification and quantitation). Recovery of acyl CoA esters was determined with each set of analyses. A standard mixture of esters was added, before extraction, to triplicate samples of powdered lyophilized rat liver. Recovery was calculated as the difference between these samples with and without the mixture of added acyl CoA standards. This difference was then compared with the same CoA standard mixture that had not been subjected to the extraction process. Recovery values for CoA compounds averaged $\sim 80 \%$, but varied from $\sim 60$ to $95 \%$ (data not shown). The appropriate value for each day and each compound was used to correct the measured acyl CoA values.

\section{Results}

The assay used for analysis of acyl CoA compounds is illustrated in Fig. 1. Each peak represented $\sim 200$ pmol of standard. The earliest compound to elute was malonyl $\operatorname{CoA}(A)$ at 


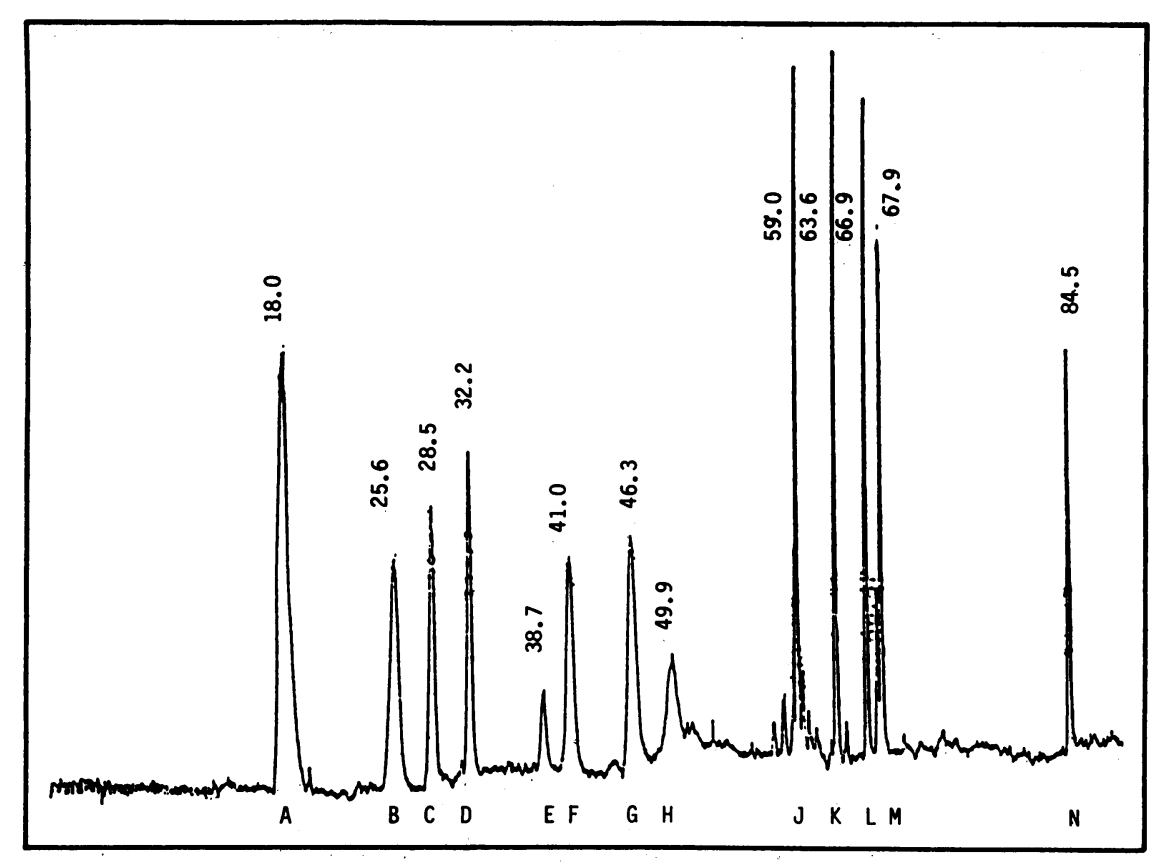

Figure 1. Elution profile of a mixture of acyl CoA standards. A $20-\mu l$ aliquot containing $\sim 200$ pmol of each standard was injected onto the Novapak $\mathrm{C}_{18}$ column and eluted using combination gradient and flow programming as described in Methods. The numbers accompanying each peak represent the time of elution in minutes. The letter identifies the compound as follows: $A$, malonyl $\mathrm{CoA} ; B$, glutathione CoA; $C$, CoASH; $D$, methylmalonyl CoA; $E$, succinyl CoA; $F, \beta$-hydroxy- $\beta$-methylglutaryl CoA; $G$, acetyl $\mathrm{CoA} ; H$, acetoacetyl CoA; J, propionyl CoA; $K$, (iso)butyryl CoA; $L, \beta$-methylcrotonyl $\mathrm{CoA} ; M$, isovaleryl CoA; and $N$, octanoyl CoA.
$18 \mathrm{~min}$ and the last was octanoyl $\operatorname{CoA}(N)$ at $84 \mathrm{~min}$. The remaining compounds, in order of elution, were glutathione CoA (B), 26 min; CoASH (C), 29 min; methylmalonyl CoA $(D), 32$ min; succinyl CoA (E), 39 min; $\beta$-hydroxy- $\beta$-methylglutaryl CoA $(F), 41$ min; acetyl CoA $(G), 46$ min; acetoacetyl CoA $(H), 50 \mathrm{~min}$; propionyl $\mathrm{CoA}(J), 60 \mathrm{~min}$; (iso)butyryl CoA $(K), 64$ min; $\beta$-methylcrotonyl CoA $(L), 67 \mathrm{~min}$; and isovaleryl $\mathrm{CoA}(M), 68 \mathrm{~min}$. Since butyryl $\mathrm{CoA}$ and isobutyryl CoA were not completely separated in most analyses, the combined peaks are referred to as (iso)butyryl CoA.

A more rapid separation of esters with earlier elution times was not feasible, due to interference from abundant absorbing material that eluted during the first $15 \mathrm{~min}$. This is illustrated in the chromatogram of Fig. 2 for a typical biopsy sample representing $\sim 20 \mathrm{mg}$ (wet weight) of liver. Note the elution of numerous peaks during the early portion of the chromatogram that were mainly adenine and pyridine nucleotides. Separation and identification of the early eluting adenine and pyridine nucleotide peaks requires a longer elution period in straight buffer without acetonitrile (18), which was not done in these studies. The large, unidentified peak at $58 \mathrm{~min}$ probably represented flavin nucleotides, since it coeluted with a flavin nucleotide standard (data not shown) and was retained after alkaline hydrolysis $(19,20)$.

The individual acyl CoA values from 10 human subjects and the thean values for each of the three patient groups, are shown in Table III. The data, recalculated to show the percentage distribution in each group, are shown in Table IV. Each group had a characteristic patterns of acyl CoA distribution. The control profile consisted of $\sim 60 \%$ free CoASH, followed by acetyl and succinyl CoA with $\sim 15$ and $14 \%$ of the total pool, respectively. The absolute level of acetyl CoA, which is

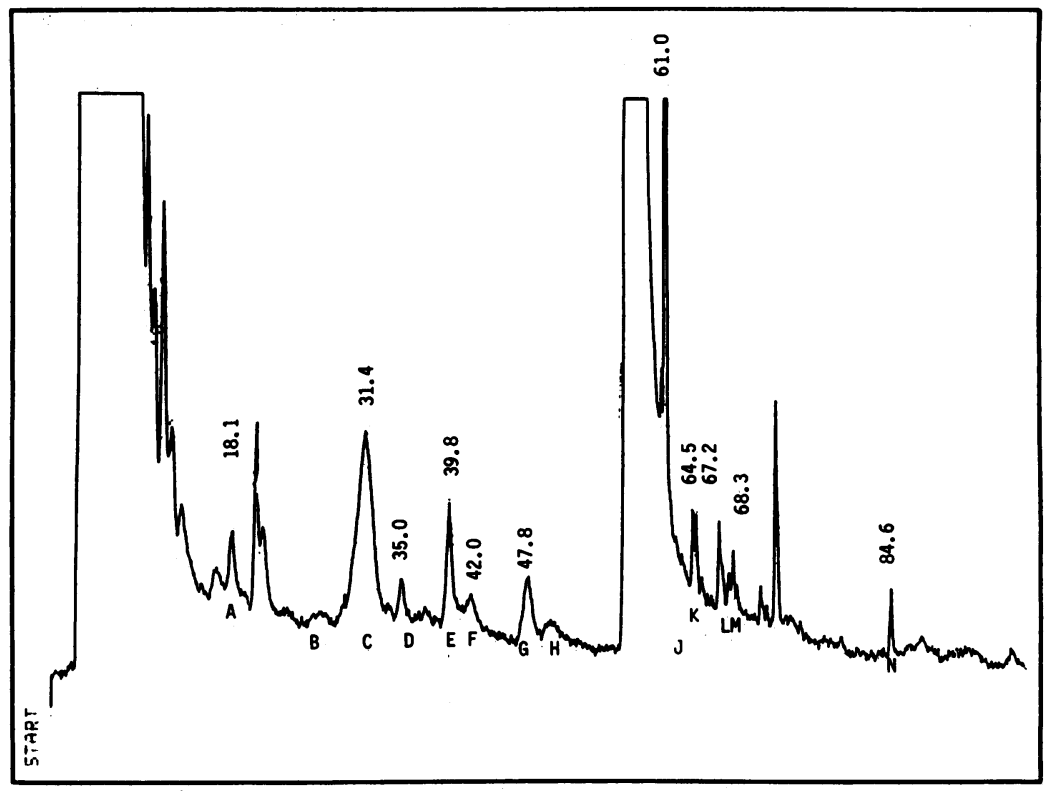

Figure 2. Illustration of an elution profile of a liver biopsy sample. A $20-\mu 1$ aliquot of sample extract, representing $\sim 20 \mathrm{mg}$ of liver wet weight was injected onto the Novapak $\mathrm{C}_{18}$ column and eluted as described in Methods. The numbers accompanying each peak represent the time of elution in minutes. The letter identifies the compound as described in the legend to Fig. 1. Letters have been placed where designated peaks are expected to elute in the case of glutathione $\operatorname{CoA}(B)$ and acetoacetyl $\operatorname{CoA}(H)$. The sample was selected for illustration only. 
Table III. Acyl CoA Profiles in Human Liver Biopsy Samples

\begin{tabular}{|c|c|c|c|c|c|c|c|c|c|c|c|c|c|}
\hline \multirow[b]{2}{*}{ CoA compound } & \multicolumn{6}{|c|}{ Control } & \multicolumn{3}{|c|}{ MCAD } & \multicolumn{4}{|c|}{ RS } \\
\hline & C-1 & $\mathrm{C}-2$ & C-3 & $C-4$ & C.5 & Mean \pm SD & M-1 & M-2 & Mean & R-1 & R-2 & $\mathbf{R}-\mathbf{3}$ & Mean \pm SD \\
\hline & \multicolumn{13}{|c|}{ nmol/g wet weight } \\
\hline CoASH & 152 & 200 & 104 & 144 & 169 & $154 \pm 35$ & 145 & 116 & 131 & 13 & 11 & 8.4 & $11 \pm 1.8^{*}$ \\
\hline Acetyl & 32 & 41 & 23 & 21 & 97 & $43 \pm 31$ & 7.2 & 8.6 & 7.9 & 32 & 29 & 12 & $24 \pm 8.8$ \\
\hline Methylmalonyl & $<2$ & $<2$ & $<2$ & $<2$ & $<2$ & $<2$ & 7.6 & 9.7 & 8.7 & 13 & 12 & 9.2 & $11 \pm 1.9$ \\
\hline Succinyl & 14 & 46 & 9.3 & 42 & 100 & $42 \pm 36$ & 30 & 46 & 38 & $<1$ & 1.9 & 8.5 & $3.7 \pm 3.5$ \\
\hline $\mathrm{HMG}^{\ddagger}$ & 4.3 & 2.3 & 70 & 9.1 & 23 & $22 \pm 27$ & $<2$ & $<2$ & $<2$ & $<1$ & $<1$ & $<1$ & $<1$ \\
\hline Propionyl & $<2$ & $<2$ & $<2$ & $<2$ & $<2$ & $<2$ & - & - & - & 0.4 & 11 & 8.0 & $6.4 \pm 5.4^{*}$ \\
\hline (Iso)butyryl & $<2$ & $<2$ & $<2$ & $<2$ & $<2$ & $<2$ & - & - & - & 8.4 & 12 & 6.3 & $8.8 \pm 2.8^{*}$ \\
\hline Isovaleryl & $<2$ & $<2$ & $<2$ & $<2$ & $<2$ & $<2$ & - & - & - & 15 & 17 & 14 & $16 \pm 1.6^{*}$ \\
\hline Octanoyl & $<2$ & $<2$ & $<2$ & $<2$ & $<2$ & $<2$ & - & - & - & 8.9 & 8.7 & 8.6 & $8.7 \pm 0.2^{*}$ \\
\hline Total soluble & 202 & 290 & 207 & 216 & 388 & $261 \pm 71$ & 190 & 180 & 185 & 90 & 103 & 75 & $89 \pm 11^{*}$ \\
\hline
\end{tabular}

Individual values and means \pm SD for five control (C) subjects; two patients with MCAD deficiency; and three patients with Reye syndrome (RS). - , an assay was not performed for the indicated metabolite. 10-25 mg wet weight human liver biopsy samples were extracted and analyzed as described in Methods. * Value differed significantly from human control values according to the unpaired $t$ test $(P<0.05)$. ${ }^{\ddagger}$ HMG, $\beta$-hydroxy- $\beta$-methylglutaryl.

proportional to the rate of fatty acid oxidation, varied as expected with respect to the nutritional state from 20 to 40 $\mathrm{nmol} / \mathrm{g}$, in the fed controls, to $\sim 100 \mathrm{nmol} / \mathrm{g}$ in the fasted subject. The ratio of free CoASH to acetyl CoA, a measure inversely proportional to the rate of fatty acid oxidation, was $\sim 4$ in control subjects. In one control patient, a very high level of $\beta$-hydroxy- $\beta$-methylglutaryl CoA $(70 \mathrm{nmol} / \mathrm{g})$ was found; this was apparently a pathological finding. None of the three human groups showed detectable levels of $\beta$-methylcrotonyl, $\beta$-methylglutaconyl, or tiglyl CoA.

In the two patients with MCAD deficiency, the levels of both free CoASH and succinyl CoA were similar to control subjects. However, acetyl CoA levels were markedly depressed, representing only $4 \%$ of the total pool. As a result, the ratio of CoASH to acetyl CoA rose fourfold from control levels to 16. Compared with control subjects, MCAD patients showed an increase in methylmalonyl CoA, representing $\sim 5 \%$ of the total pool. Several acyl CoA compounds, including octanoyl CoA, were not measured in these patients. ${ }^{3}$

The acyl CoA profiles of the three Reye syndrome patients were distinct from the other two groups. Unlike control subjects, these patients accumulated unusual CoA esters, including methylmalonyl, propionyl, (iso)butyryl, isovaleryl, and octanoyl CoA. In these patients, the absolute levels of acetyl CoA, averaging $24 \mathrm{nmol} / \mathrm{g}$ were about half the control value. Still, acetyl CoA was the major metabolite, representing $\sim 25 \%$ of the total pool. Free CoASH levels were dramatically reduced in these patients to $\sim 11 \mathrm{nmol} / \mathrm{g}$, or $<10 \%$ of the control levels. Largely due to the drop in free $\mathrm{CoASH}$, the ratio of free CoASH to acetyl CoA fell to $\sim 0.5$, or $10 \%$ of the control value.

3. MCAD liver biopsy samples were analyzed before identification of the enzyme deficiency and before expansion of the assay to include octanoyl CoA. Subsequent studies on fibroblasts obtained from these patients, incubated in media containing FFA demonstrated accumulation of octanoyl CoA.
The Reye syndrome patients also had a dramatic reduction in the total soluble pool of short- and medium-chain acyl CoA esters. The average size of the pool was $\sim 90 \mathrm{nmol} / \mathrm{g}$, or onethird of control values. The method did not distinguish between a decrease caused by a drop in the combined acid-soluble and -insoluble pools, or by an increase of CoA in the unmeasured insoluble pool, which contained the long-chain acyl CoA metabolites.

Table IV compares the percent distribution of CoA intermediates between the rat and human groups. Throughout, the data for rats and control humans were remarkably similar. The total amount of acyl CoA esters varied with nutritional state in both human subjects and rats. The human subjects, who were generally in a well-nourished state, showed CoA profiles resembling fed rather than starved rats. This was the case in all but one of the controls, C-5. Unlike the other human controls, this 6-yr-old was fasted overnight and had an acyl CoA profile resembling that of the fasted rats. A number of minor metabolites were measured in the rat that were not detected in the human control groups. Most likely, this was due to the abundance of rat tissue available for biochemical assay, not to intrinsic metabolic differences.

\section{Discussion}

This study demonstrated, for the first time, the measurement of acyl CoA esters in human liver biopsy samples. Use of the method to probe fatty acid oxidation in human subjects offers several advantages over previous techniques. First, since the biopsy samples were quickly frozen, the tissue underwent far less cellular autolysis than in a previous postmortem study (16). The method also permits identification of specific shortand medium-chain acyl CoA compounds, whereas prior techniques measured the entire pool $(16,20)$. The biopsy method also can be used to study earlier stages of the disease, providing insight into the progression of the pathology.

The study also showed a striking similarity between rat and 
Table IV. Percent Distribution of Acyl CoA Compounds in Human and Rat Liver

\begin{tabular}{|c|c|c|c|c|c|}
\hline \multirow[b]{2}{*}{ CoA compound } & \multirow[b]{2}{*}{ Human controls } & \multirow[b]{2}{*}{ Reye syndrome } & \multirow[b]{2}{*}{ MCAD } & \multicolumn{2}{|c|}{ Rat liver } \\
\hline & & & & Fed & Starved \\
\hline & \multicolumn{5}{|c|}{$\%$} \\
\hline CoASH & $60 \pm 13$ & $12 \pm 1.9^{*}$ & 70 & $55 \pm 5^{\ddagger}$ & $48 \pm 4^{\ddagger}$ \\
\hline Acetyl & $15 \pm 5.9$ & $26 \pm 9.7$ & 4.3 & $10 \pm 1$ & $28 \pm 6$ \\
\hline$(\text { CoASH/Acetyl })^{8}$ & $(4.0)$ & $(0.5)$ & (17) & $(5.5)$ & (1.8) \\
\hline Methylmalonyl & $<1$ & $13 \pm 1.4^{*}$ & 4.7 & $4.6 \pm 1.0^{\ddagger}$ & $1.5 \pm 2.0^{\ddagger}$ \\
\hline Succinyl & $14 \pm 8.9$ & $4.4 \pm 6.1$ & 21 & $6.0 \pm 1.7$ & $22 \pm 5$ \\
\hline HMG & $9.4 \pm 14$ & $<1$ & $<1$ & $7.8 \pm 1.8$ & $2.8 \pm 0.6$ \\
\hline Propionyl & $<1$ & $7.2 \pm 5.9^{*}$ & - & $12 \pm 3^{*}$ & $<0.5$ \\
\hline (Iso)butyryl & $<1$ & $9.7 \pm 1.8^{*}$ & - & $1.3 \pm 0.4^{\ddagger}$ & $<0.5^{\ddagger}$ \\
\hline Isovaleryl & $<1$ & $18 \pm 1.2^{*}$ & - & $0.6 \pm 0.1^{\ddagger}$ & $0.5 \pm 0.6^{\ddagger}$ \\
\hline Octanoyl & $<1$ & $9.9 \pm 1.9^{*}$ & - & $1.4 \pm 0.4^{\ddagger}$ & $<0.5^{\ddagger}$ \\
\hline Total soluble CoA & 261 & 89 & 185 & 231 & 386 \\
\hline
\end{tabular}

Values for human livers were calculated from the data in Table III. Values of the individual CoA metabolites \pm SD are expressed as mean percentages of the total acid-soluble acyl CoA pool. Rat liver data were obtained from 50-mg aliquots of freeze-clamped, powdered samples from fed or 48-h-fasted Sprague-Dawley rats. Each value is an average from 3-5 rats. Total soluble CoA is the mean value expressed in nanomoles per gram wet weight. *Value differs significantly from human controls $(P<0.05)$. ${ }^{\ddagger}$ Value differs significantly from Reye syndrome patients $(P<0.05) .{ }^{\S}$ Values in parentheses represent ratios of CoASH/Acetyl CoA.

human acyl CoA profiles, suggesting that the rat may be a useful animal model for investigation of Reye syndrome. So far, efforts to study the disease have been hampered by lack of an animal model in which to test hypotheses of pathogenesis (2). A rat model also would permit correlation of biochemical and morphological events.

Measured levels of acyl CoA compounds supported the hypothesis that fatty acid oxidation is compromised in Reye syndrome patients $(2,7)$. The unusual short- and mediumchain acyl CoA esters that accumulated in the livers are likely precursors of the deacylated compounds previously reported in the blood and urine of these patients (2-7).

At least three explanations are possible for the unusual acyl CoA profiles in these patients. One possibility is a generalized increase in proteolysis. Protein breakdown would lead to increased production of branched-chain amino and ketoacids, precursors to several of the compounds observed in this study, including isovaleryl, isobutyryl, propionyl, and methylmalonyl $\operatorname{CoA}(10,17)$. Contradicting the occurrence of widespread proteolysis was the failure to detect a generalized increase in other metabolites of branched-chain ketoacid oxidation.

Alternatively, a multiple acyl CoA dehydrogenation defect (glutaric acid-uria type II), caused by deficiency of electron transfer flavoprotein or electron transfer flavoprotein dehydrogenase, could account for the unusual organic aciduria (21). This disorder is characterized by accumulation and excretion of organic acids derived from acyl CoA substrates of mitochondrial acyl CoA dehydrogenases. However, additional compounds that are not substrates of these enzymes also appear in the urine of patients with Reye syndrome.

The final, and most likely hypothesis is a multiple-site inhibition of the mitochondrial enzymes involved in fatty acid and branched-chain amino acid metabolism. Morphological and biochemical evidence support this concept. The pleomorphic mitochondria found in Reye syndrome patients show disrupted cristae (22), sites of the membrane-associated acyl CoA dehydrogenases of both branched-chain and fatty acid oxidation $(23,24)$. A generalized mitochondrial defect also is consistent with the diversity of accumulated metabolites, suggesting that other enzymes besides acyl CoA dehydrogenases may be impaired. For example, the accumulation of propionyl and methylmalonyl CoA esters indicates defects in propionyl CoA carboxylase or methylmalonyl CoA mutase (10-12). It is not known if these mitochondrial enzymes are membrane associated.

Given the evidence for impaired fatty acid oxidation in Reye syndrome, the near normal levels of acetyl CoA in these patients were surprising. One explanation for this puzzle may be increased production of cytosolic acetyl CoA. Acetyl CoA concentration gradients between mitochondria and cytosol vary from 6.6 to 25 in hepatocytes from fed rats, depending on substrate (25). While mitochondrial fatty acid oxidation is inhibited, alternative pathways thus may have stepped up production of acetyl CoA. If so, peroxisomes would be likely sites for this extramitochondrial activity, since these organelles oxidize fatty acids $(8,9)$ and proliferate in Reye syndrome $(22)$. Fatty acid oxidation by peroxisomes also is limited to substrates of chain length $\geqq 8(8,9)$, consistent with the absence of long-chain acyl CoA ester accumulation in patients with Reye syndrome (16). Other evidence for a peroxisomal pathway is the abnormally high levels of acetyl carnitine, a presumed end product of peroxisomal fatty acid oxidation, excreted by these patients $(26,27)$.

Alternatively, acetyl CoA might accumulate to near normal levels due to blockade of its conversion to acetoacetate. However, no intermediates of the ketogenic pathway were detected, a finding consistent with the known low rate of ketogenesis in Reye syndrome.

The results of this study are summarized in the model shown in Fig. 3. We postulate that mitochondrial $\beta$-oxidation 


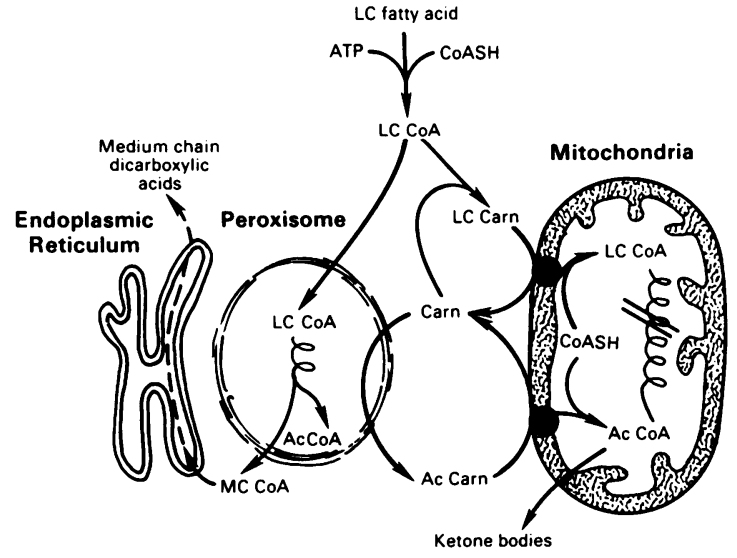

Figure 3. Model illustrating interactions between mitochondrial $\beta$ oxidation, peroxisomal $\beta$-oxidation, and $\omega$-oxidation in the endoplasmic reticulum. The model shows oxidation of activated long-chain (LC) fatty acids by both mitochondria and peroxisomes. The end product of mitochondrial $\beta$-oxidation is primarily ketone bodies, whereas peroxisomal $\beta$-oxidation yields acetyl carnitine (Ac Carn) and medium chain (MC) acyl CoA esters. $\omega$-Oxidation converts these MC intermediates to dicarboxylic acids. A putative block in mitochondrial $\beta$-oxidation would increase fatty acid oxidation by peroxisomal and endoplasmic reticular pathways.

is blocked at multiple acyl CoA dehydrogenases, resulting in accumulation of short-, medium-, and long-chain acyl CoA compounds. Since short- and some medium-chain acyl CoA esters are not substrates for peroxisomal $\beta$-oxidation $(8,9)$, they would either be degraded in the microsomes by $\omega$-oxidation or deacylated and excreted. Long-chain fatty acyl CoA esters would not accumulate in this scheme, but instead would be oxidized in the peroxisomes. That would explain why acetyl carnitine appears in the urine, but ketone bodies, the end products of mitochondrial fatty acid oxidation, do not accumulate normally $(2,27)$.

The model also could explain findings in disorders of fatty acid metabolism in patients with long-chain acyl CoA dehydrogenase or MCAD deficiency. In long-chain acyl CoA dehydrogenase deficiency the first enzyme of mitochondrial $\beta$-oxidation is defective (28). Such patients excrete acetyl carnitine in the urine, consistent with the idea that peroxisomal $\beta$-oxidation is involved. Medium-chain dicarboxylic acids are also excreted by these patients. The model would predict that these compounds are derived from accumulated medium-chain acyl CoA esters, end products of peroxisomal $\beta$-oxidation, which are subsequently degraded by $\omega$-oxidation to dicarboxylic acids. In patients with MCAD deficiency, a partial block of mitochondrial $\beta$-oxidation would result in diminished acetyl CoA levels, as shown in this study, and decreased ketone body levels, as reported previously (13). Because of the block, mitochondrial fatty acid oxidation can proceed only to mediumchain fatty acyl CoA esters. Since these compounds cannot be further degraded by the peroxisomes, they would be metabolized and excreted as medium-chain acyl carnitines and dicarboxylic acids. This idea is consistent with the reported presence of octanoyl carnitine and dicarboxylic acids in the urine of MCAD patients $(29,30)$.

The findings also provide potential mechanisms for several major abnormalities observed in Reye syndrome. The hyper- ammonemia found in these patients (1-5) could result from the observed accumulation of methylmalonyl and propionyl CoA esters, since these compounds inhibit two key enzymes in ureogenesis, carbamyl phosphate synthetase I and $N$-acetylglutamate synthetase (10-12). Likewise, the hypoglycemia observed in Reye syndrome (1-5) could result from the accumulation of methylmalonyl $\mathrm{CoA}$, since it inhibits pyruvate carboxylase (10-12), the first and rate-limiting enzyme in gluconeogenesis. Finally, the very low ratios of CoASH/acetyl CoA observed in Reye syndrome patients could inhibit mitochondrial energy generation $(10-12,31,32)$. Flux through pyruvate dehydrogenase and $\alpha$-ketoglutarate dehydrogenase, two enzymes involved in ATP production, are regulated by CoASH levels. High levels of propionyl $\mathrm{CoA}$ and methylmalonyl CoA also could inhibit citrate synthase, another Krebs cycle enzyme (10-12). Since both ureogenesis and gluconeogenesis require mitochondrial ATP, secondary inhibition of these pathways could also result from energy deficiency.

Still to be determined is the trigger that initiates this cascade of events in Reye syndrome. A genetic defect, viral infection, salicylate toxicity, and endotoxin are among the factors suggested as possible causes of the disease (33-39). One intriguing candidate is tumor necrosis factor (TNF). TNF production follows viral infection or endotoxin stimulation (35, 38), conditions that also precede Reye syndrome. Moreover, the ingestion of aspirin, which also stimulates TNF production (35), usually precedes the onset of the disease. Whatever the causative factor, the findings in this study put constraints on future models, which must account for the unusual pattern of acyl CoA esters found in Reye syndrome.

\section{Acknowledgments}

The authors express gratitude to Dr. Jean Wallace for generous and excellent editorial assistance.

This work was supported by National Institutes of Health grants NS-17752, DK-35914, DK-19525, RR-00240, and HL-26895.

\section{References}

1. Reye, B. D. K., G. Morgan, and J. Baral. 1963. Encephalopathy and fatty degeneration of the viscera: a disease entity in childhood. Lancet. ii:749-751.

2. Heubi, J. E., J. C. Partin, J. S. Partin, and W. K. Schubert. 1987. Reye's syndrome: current concepts. Hepatology (Baltimore). 7:155164.

3. DeVivo, D. C. 1985. Reye syndrome. Neurol. Clin. N. Am. 3:95-115.

4. 1985. Reye's Syndrome-United States, 1984. JAMA (J. Am. Med. Assoc.). 253:751-753.

5. Brown, R. E., and D. T. Forman. 1982. The biochemistry of Reye's syndrome. CRC Crit. Rev. Clin. Lab. Sci. 17:247-297.

6. Trauner, D. A., W. L. Nyhan, and L. Sweetman. 1975. Shortchain organicacidemia and Reye's syndrome. Neurology. 25:296-298.

7. Tonsgard, J. H. 1985. Urinary dicarboxylic acids in Reye syndrome. J. Pediatr. 107:79-84.

8. Lazarow, P. B., and C. DeDuve. 1976. A fatty acyl CoA oxidizing system in rat liver peroxisomes: enhancement by clofibrate, a hypolipidemic drug. Proc. Natl. Acad. Sci. USA. 73:2043-2046.

9. Lazarow, P. B. 1978. Rat liver peroxisomes catalyze the $\beta$ oxidation of fatty acids. J. Biol. Chem. 253:1522-1528.

10. Corkey, B. E., A. Martin-Requero, E. Walajtys-Rode, R. J. Williams, and J. R. Williamson. 1982. Regulation of the branched chain $\alpha$-ketoacid pathway in liver. J. Biol. Chem. 257:9668-9676. 
11. Martin-Requero, A., B. E. Corkey, S. Cerdan, E. WalajtysRode, R. Parrilla, and J. R. Williamson. 1983. Interactions between $\alpha$-ketoisovalerate metabolism and the pathways of gluconeogenesis and urea synthesis in isolated hepatocytes. J. Biol. Chem. 258:36733681.

12. Williamson, J. R., B. E. Corkey, A. Martin-Requero, and K. E. Coll. 1986. Metabolic repercussions of branched chain ketoacid metabolism in liver. In Branched Chain Amino Acids in Physiology and Medicine. R. Odessey, editor. Elsevier/North Holland, New York. 135-172.

13. Stanley, C. A., D. E. Hale, P. M. Coates, C. L. Hall, B. E. Corkey, W. Yang, R. I. Kelley, E. L. Gonzales, J. R. Williamson, and L. Baker. 1983. Medium chain acyl CoA dehydrogenase activity in children with non-ketotic hypoglycemia and low carnitine levels. $\mathrm{Pe}$ diatr. Res. 17:877-884.

14. Coates, P. M., D. E. Hale, C. A. Stanley, B. E. Corkey, and J. Cortner. 1985. Genetic deficiency of medium chain acyl CoA dehydrogenase studied in cultured skin fibroblasts and peripheral mononuclear leukocytes. Pediatr. Res. 19:672-676.

15. Stanley, C. A., and P. M. Coates. 1985. Inherited defects of fatty acid oxidation which resemble Reye syndrome. J. Nat. Reye's Synd. Fndn. 5:190-200.

16. Kang, E. S., M. T. Capaci, D. N. Korones, and N. Tekade. 1982. Liver coenzyme A ester content: comparison between Reye's syndrome and control subjects. Clin. Sci. 63:455-460.

17. Corkey, B. E., M. Brandt, R. J. Williams, and J. R. Williamson. 1981. Assay of short chain acyl CoA intermediates in tissue extracts by high pressure liquid chromatography. Anal. Biochem. 118:30-41.

18. Brown, P. R., and A. M. Krstulovic. 1979. Practical aspects of reversed-phase liquid chromatography applied to biochemical and biomedical research. Anal. Biochem. 99:1-21.

19. Williamson, J. R., and B. E. Corkey. 1969. Assays of intermediates of the citric acid cycle and related compounds by fluorimetric enzyme methods. Methods Enzymol. 13:434-513.

20. Williamson, J. R., and B. E. Corkey. 1979. Assay of intermediates of the citric acid cycle and related compounds: update with tissue metabolite levels and intracellular distribution. Methods Enzymol. 55:200-222.

21. Frerman, F. E., and S. I. Goodman. 1985. Deficiency of electron transfer flavoprotein or electron transfer flavoprotein:ubiquinone oxidoreductase in glutaric acidemia Type II fibroblasts. Proc. Natl. Acad. Sci. USA. 82:4517-4520.

22. Partin, J. C., W. K. Schubert, and J. S. Partin. 1971. Mitochondrial ultrastructure in Reye's syndrome (encephalopathy and fatty degeneration of the viscera). N. Engl. J. Med. 285:1339-1343.

23. Ikeda, Y., C. A. Dabrowski, and K. Tanaka. 1983. Separation and properties of five distinct acyl $\mathrm{CoA}$ dehydrogenases from rat liver mitochondria: identification of a new 2-methyl branched chain acyl CoA dehydrogenase. J. Biol. Chem. 258:1066-1076.

24. Ikeda, Y., and K. Tanaka. 1983. Purification and characterization of 2-methyl branched chain acyl CoA dehydrogenase, an enzyme involved in isoleucine and valine metabolism, from rat liver mitochondria. J. Biol. Chem. 258:9477-9487.
25. Siess, E. A., D. G. Brocks, and O. H. Wieland. 1978. Distribution of metabolites between the cytosolic and mitochondrial compartments of hepatocytes isolated from fed rats. Hoppe-Seyler's Z. Physiol. Chem. 359:785-798.

26. Millington, D. S., C. R. Roe, and D. A. Maltby. 1984. Application of high resolution fast atom bombardment and constant $B / E$ ratio linked scanning to the identification and analysis of acyl carnitines in metabolic disease. Biomed. Mass Spectrom. 11:236-241.

27. Roe, C. R., D. S. Millington, D. A. Maltby, and T. P. Bohan. 1985. J. Nat. Reye's Synd. Fndn. 5:201-219.

28. Hale, D. E., M. L. Batshaw, P. M. Coates, F. E. Frerman, F. E. Goodman, I. Singh, and C. A. Stanley. 1985. Long chain acyl CoA dehydrogenase deficiency: an inherited cause of nonketotic hypoglycemia. Pediatr. Res. 19:666-671.

29. Duran, M., G. Mitchell, J. B. C. de Klerk, J. P. de Jager, M. Hofkamp, L. Bruinvis, D. Ketting, J.-M. Saudubray, and S. K. Wadman. 1985. Octanoic acidemia and octanylcarnitine excretion with dicarboxylic aciduria due to defective oxidation of medium-chain fatty acids. J. Pediatr. 107:397-404.

30. Roe, C. R., D. S. Millington, D. A. Maltby, and P. Kinnebrew. 1986. Recognition of medium-chain acyl-CoA dehydrogenase deficiency in asymptomatic siblings of children dying of sudden infant death or Reye-like syndromes. J. Pediatr. 108:13-18.

31. Walajtys-Rode, E., K. E. Coll, and J. R. Williamson. 1979. Effects of branched chain $\alpha$-ketoacids on the metabolism of isolated rat liver cells. 1. Regulation of branched chain $\alpha$-ketoacid metabolism. $J$. Biol. Chem. 254:11511-11520.

32. Walajtys-Rode, E., and J. R. Williamson. 1980. Effects of branched chain $\alpha$-ketoacids on the metabolism of isolated rat liver cells. III. Interaction with pyruvate dehydrogenase. J. Biol. Chem. 255:413-418.

33. Trauner, D. A., M. Donoviel, and L. E. Davis. 1983. Effects of influenza $B$ virus on mouse mitochondrial $\beta$-oxidation of fatty acids: implications for Reye's syndrome. Neurology. 33:88-98.

34. Starko, K. M., G. C. Ray, L. B. Domingues, W. L. Stromberg, and D. F. Woodall. 1980. Reye's syndrome and salicylate use. Pediatrics. 66:859-865.

35. Larrick, J. W., and S. L. Kunkel. 1986. Is Reye's syndrome caused by augmented release of tumor necrosis factor? Lancet. ii:132133.

36. Yoder, M. C., J. M. Egler, M. Yudkoff, J. Chatten, S. D. Douglas, and R. A. Polin. 1985. Metabolic and mitochondrial morphological changes that mimic Reye syndrome after endotoxin administration to rats. Infect. Immun. 47:329-331.

37. Kilpatrick-Smith, L., S. D. Douglas, R. A. Polin, and B. E. Corkey. 1987. Effects of sublethal doses of endotoxin and aspirin on rat hepatic energy metabolism: an animal model of Reye syndrome. Pediatr. Res. 21:343. (Abstr.)

38. Kilpatrick-Smith, L., S. D. Douglas, and B. E. Corkey. 1987. Effects of monokines on calcium signal transduction in isolated hepatocytes and neuroblastoma cells. Circ. Shock. 21:311a. (Abstr.)

39. Douglas, S. D., L. Kilpatrick-Smith, R. A. Polin, G. B. Kolski, and Y. Levy. 1987. Serum acid proteolytic activity in Reye syndrome. Lancet. i:221. 